\title{
Response to: Tyrosine 397 phosphorylation is critical for FAK-promoted Rac1 activation and invasive properties in oral squamous cell carcinoma cells
}

Laboratory Investigation (2016) 96, 1027; doi:10.1038/labinvest.2016.81

I thank the editors for the opportunity to respond to Dr Sylow's letter. In the letter, Dr Sylow notes potential concerns that therapeutic administration of Racl inhibitors for patients with metastatic disease may result in adverse effects of insulin resistance and hyperglycemia, thus promoting the development of type 2 diabetes.

Despite no Racl inhibitor currently available in clinical, I sincerely agree the points raised by Dr Sylow and the detailed evaluation or monitoring is critical for clinical application of Rac1 inhibitors in the future. However, there are two issues which need to be discussed. First, Rac1 inhibition causing insulin resistance and hyperglycemia is based on in vitro and in vivo studies. Whether the consistent results are also found in clinical trials requires further investigation. It is because the consequence will be closely associated with the doses or periods of treatment and the diversity of patients' conditions. This notion is supported by the clinical studies that the majority of inhibitors which target the PI3K/AKT/mTOR pathway, the key pathway of insulin signaling, cause a range from 7 to $50 \%$ cancer patients developing hyperglycemia. ${ }^{1}$ Treatment of everolimus, an approved mTOR inhibitor, leads to hyperglycemia in 50\% of patients with advanced renal cell carcinoma but only in $13 \%$ of patients with breast cancer. In addition, hyperglycemia is reported in $26 \%$ of all grade RCC patients who receive temsirolimus, the other approved mTOR inhibitor, whereas grades 3-4 hyperglycemic events were less frequency $(11 \%){ }^{2}$

Second, the goal of treatment in patients with metastatic disease is to save the life and to preserve the quality of life. If the Rac1 inhibitors effectively suppress cancer spreading, it is worthy to know whether Racl inhibition-caused insulin resistance or hyperglycemia can be alleviated by specific management. Since targeted therapy drugs, including mTOR inhibitors (everolimus and temsirolimus) and tyrosine-kinase inhibitors (nilotinib, pazopanib and sunitinib), have been reported to cause hyperglycemia or hyperlipidemia, ${ }^{2}$ the medical management proposed by a Task Force of the US National Cancer Institute Investigation Drug Steering Committee $^{1}$ may provide the guideline for dealing the metabolic complications caused by anti-cancer therapy such as Rac1 inhibitors.

In summary, we believe our previous results supporting the notion that Racl can serve as the therapeutic target for treating patients with metastatic OSCC. Despite the existence of potential adverse effects, we can learn the lesson from the clinical application of PI3K/AKT/mTOR inhibitors and use these lifesaving anti-cancer therapies appropriately with the careful monitoring and management.

Ta-Chun Yuan

Department of Life Science, National Dong Hwa University, Hualien, Taiwan E-mail: yuan415@mail.ndhu.edu.tw

\section{DISCLOSURE/CONFLICT OF INTEREST}

The author declares no conflict of interest.

1. Busaidy NL, Farooki A, Dowlati A et al. Management of metabolic effects associated with anticancer agents targeting the PI3K-Akt-mTOR pathway. J Clin Oncol 2012;30:2919-2928.

2. Vergès $B$, Walter $T$, Cariou B. Endocrine side effects of anti-cancer drugs: effects of anti-cancer targeted therapies on lipid and glucose metabolism. Eur J Endocrinol 2014;170:R43-R55. 